\title{
Assessment of Water Quality Modelling Capabilities of EPANET Multiple Species and Pressure-Dependent Extension Models
}

\author{
Alemtsehay G. Seyoum, Tiku T. Tanyimboh and Calvin Siew \\ Department of Civil Engineering, University of Strathclyde Glasgow, \\ John Anderson Building, 107 Rottenrow, Glasgow G4 0NG, UK \\ (Email: alemtsehay.seyoum@strath.ac.uk,tiku.tanyimboh@strath.ac.uk,calvin.siew@strath.ac.uk)
}

This article was published in Water Science and Technology: Water Supply.

The final publication is available at: http://ws.iwaponline.com/content/13/4/1161.

Assessment of water quality modelling capabilities of EPANET multiple species and pressure-dependent extension models

Alemtsehay G. Seyoum, Tiku T. Tanyimboh, Calvin Siew

Water Science and Technology: Water Supply (2013), 13(4), pages 1161-1166, DOI: 10.2166/ws.2013.118 


\title{
Assessment of Water Quality Modelling Capabilities of EPANET Multiple Species and Pressure Dependent Extension Models
}

\author{
Alemtsehay G. Seyoum, Tiku T. Tanyimboh and Calvin Siew \\ Department of Civil Engineering, University of Strathclyde Glasgow, \\ John Anderson Building, 107 Rottenrow, Glasgow G4 0NG, UK \\ (Email: alemtsehay.seyoum@strath.ac.uk,tiku.tanyimboh@strath.ac.uk,calvin.siew@strath.ac.uk)
}

\begin{abstract}
The need for accurately predicting water quality through models has increasingly been crucial in meeting rigorous standards and customer expectations. There are several endeavours on developing robust water quality models for water distribution systems. In this paper, two variants of the EPANET 2 water quality model have been assessed to inform future research. The models are the multiple species extension EPANET-MSX and the pressure dependent extension EPANET-PDX. Water quality analysis was conducted on a hypothetical network considering various operating pressure conditions. Different kinetic models were employed to simulate water quality. First order, limited first order and zero order models were used for predicting chlorine residual, disinfection by-products and water age respectively. Generally, EPANET-MSX and EPANET-PDX provided identical water quality results for normal operating conditions with adequate pressure but different results for pressure deficient networks. Also, a parallel first order model with fast and slow reacting components was used for chlorine decay and disinfection by-products using the EPANETMSX model for a network operating under normal pressure conditions.
\end{abstract}

Keywords

EPANET, pressure-deficient water distribution system, water quality modelling, disinfection by-products

\section{INTRODUCTION}

Research indicates that the quality of water in water distribution systems (WDSs) may deteriorate due to several changes that take place during transport in the distribution system (Rossman, 1993). These changes include: loss of disinfection residuals that can lead to bacterial re-growth (Clark and Haught, 2005); formation of potentially carcinogenic disinfection by-products (DBPs) due to the reactions of the disinfectant with organic and inorganic substances in water (Rodriguez et al., 2004); development of taste and odour; and corrosion. The water quality concerns in combination with the rigorous standards set by regulatory bodies have pressed water companies to depend increasingly on models in the quest to understand and control the dynamics of water quality processes.

Several computer models are available to simulate water quality processes in WDSs. EPANET 2, a public domain hydraulic and water quality model, is among the most widely used. The model enables simulation of non-reactive tracer materials, chlorine decay, DBPs growth and water age (Rossman, 2000). Primarily EPANET 2 is a single species model and limited to model the dynamics of chlorine residual, trihalomethane (THM) or water age that does not permit simulation of multiple interacting species (Shang et al., 2008). The first order kinetic decay model of EPANET 2 depends on the concentration of only one reactant. The model for chlorine decay, for example, depends only on the concentration of chlorine. This prevents the chlorine decay model from including other potential reactants such as natural organic matter.

Another drawback of EPANET 2 stems from the hydraulic analysis approach used. There are two hydraulic analysis approaches, viz the traditional demand driven analysis (DDA) and pressure 
dependent analysis (PDA) (Wu et al. 2009; Tanyimboh and Templeman 2010; Siew and Tanyimboh, 2011). DDA assumes demand is satisfied in full irrespective of the network pressure. This assumption is valid only if the network performs under normal pressure conditions with adequate pressures at all nodes (Wu et al. 2009). However, in the presence of network irregularities such as pipe breaks, pump failures, temporary demand increase e.g. for fire fighting purposes, and system maintenance and repair restrictions, DDA provides unrealistic hydraulic and water quality results. By contrast, PDA based models take into account the pressure dependent nature of nodal flows and thus the models provide results that are more realistic (Chandapillai 1991; Gupta \& Bhave 1996; Tanyimboh et al. 1999). EPANET 2 is a DDA based model.

There have been great research efforts to enhance the EPANET 2 model. Shang et al. (2008) developed a multispecies extension model called EPANET-MSX. The model enables simulation of multiple chemical species in bulk water as well as the pipe wall. EPANET-MSX can assist modellers to investigate any species and chemical reactions of interest. Also, the model takes into account the reactivity of water from different sources. It maintains wholly the existing EPANET 2 capability of simulating water quality for extended periods. A recent achievement on improving EPANET 2 has also been reported in Siew and Tanyimboh (2012). The researchers have developed a pressure dependent extension known as EPANET-PDX. The model is an extension that has a logistic pressure dependent demand function (Tanyimboh and Templeman, 2010). The model has full EPANET 2 modelling functionality and can perform hydraulic, water quality and extended period simulation of a network under normal and pressure deficient conditions in a seamless way.

The aim of this paper is to compare the water quality modelling capabilities of the multispecies and pressure dependent EPANET 2 extension models to inform future research on pressure dependent water quality modelling. We used a simple network from the literature in this study.

\section{METHODS}

To evaluate the capabilities of EPANET-PDX and EPANET-MSX different water quality analyses were conducted on a simple network in Fujiwara and Ganesharajah (1993). The analyses comprised of simulation of water age, chlorine residual and THM concentrations. Various reaction rate models and network hydraulic conditions were considered. The equations used herein for the kinetic reactions include first order, limited first order and parallel first order models. The first order (Equation 1) and limited first order (Equation 2) kinetic models were used for the simulation of chlorine decay and THM concentration in bulk water respectively.

$$
\frac{d C}{d t}=-k_{b} C
$$

where $C=$ chlorine concentration; $t=$ time; $k_{b}=$ bulk water reaction rate constant.

$$
\frac{d C}{d t}=k_{b}\left(C_{L}-C\right)
$$

where $C=$ THM concentration; $t=$ time; $k_{b}=$ bulk water reaction rate constant; and $C_{L}=$ maximum THM concentration. For modelling water age, a zero order reaction is used, i.e. $d C / d t=1$. The parallel first order model in Equation 3 was used for modelling of chlorine decay. 


$$
C_{t}=C_{0}\left[\left(F \exp \left(-k_{1} t\right)+(1-F) \exp \left(-k_{2} t\right)\right]\right.
$$

where $C_{t}=$ chlorine concentration at time $t ; C_{0}=$ initial chlorine concentration; $k_{1} \& k_{2}=$ fast and slow decay rate constants; $F=$ fraction of chlorine reacting rapidly; $(1-F)=$ fraction of chlorine reacting slowly. Helbling and Van Briesen (2009) evaluated different kinetic models and concluded that the parallel first order model provided the best results among different models. The model has two components that describe rapid and slow reactions. Sohn et al. (2004) developed a two-phase THM kinetic model (Equation 4) based on a parallel first order model. The model explicitly considers chlorine decay in the reaction kinetics.

$$
T H M=C\left\{A\left(1-\exp \left(-k_{1} t\right)\right)+B\left(1-\exp \left(-k_{2} t\right)\right)\right\}
$$

where $C=$ chlorine concentration; $k_{1} \& k_{2}=$ chlorine decay rate constants as in Equation $3 ; A \& B=$ parameters of the THM model that represent the fast and slow reacting components, respectively.

For pressure dependent modelling EPANET-PDX incorporates the Tanyimboh and Templeman (2010) logistic pressure dependent demand function into the system of hydraulic equations. The function is described as follows.

$$
Q n_{i}\left(H n_{i}\right)=Q n_{i}^{r e q} \frac{\exp \left(\alpha_{i}+\beta_{i} H n_{i}\right)}{1+\exp \left(\alpha_{i}+\beta_{i} H n_{i}\right)}
$$

where $Q n_{i}$ and $H n_{i}$ are the flow and head at node $i$ respectively. $Q n_{i}^{\text {req }}$ is demand at node $i . \alpha_{i}$ and $\beta_{i}$ are parameters to be calibrated with relevant field data. In the absence of field data, Tanyimboh and Templeman (2010) suggested that default values for $\alpha_{i}$ and $\beta_{i}$ could be taken as

$$
\begin{gathered}
\alpha_{i}=\frac{-4.595 H n_{i}^{\text {des }}-6.907 H n_{i}^{\text {min }}}{H n_{i}^{\text {des }}-H n_{i}^{\min }} \\
\beta_{i}=\frac{11.502}{H n_{i}^{\text {des }}-H n_{i}^{\text {min }}}
\end{gathered}
$$

where $H n_{i}^{\text {des }}$ is the nodal head above which $Q n_{i}=Q n_{i}^{\text {req }}$ and $H n_{i}^{\text {min }}$ is nodal head below which $Q n_{i}=0$.

\section{RESULTS AND DISCUSSIONS}

The simple two-loop network shown in Figure 1 in which $\mathrm{C}_{\mathrm{HW}}$ represents the Hazen-Williams roughness coefficient was selected for demonstration purposes (Fujiwara and Ganesharajah 1993). The network consists of a single source, 8 pipes of length $1000 \mathrm{~m}$ and 6 demand nodes. The required residual head for all nodes is $15 \mathrm{~m}$. 24-hour extended period water quality simulations of the network were conducted. 


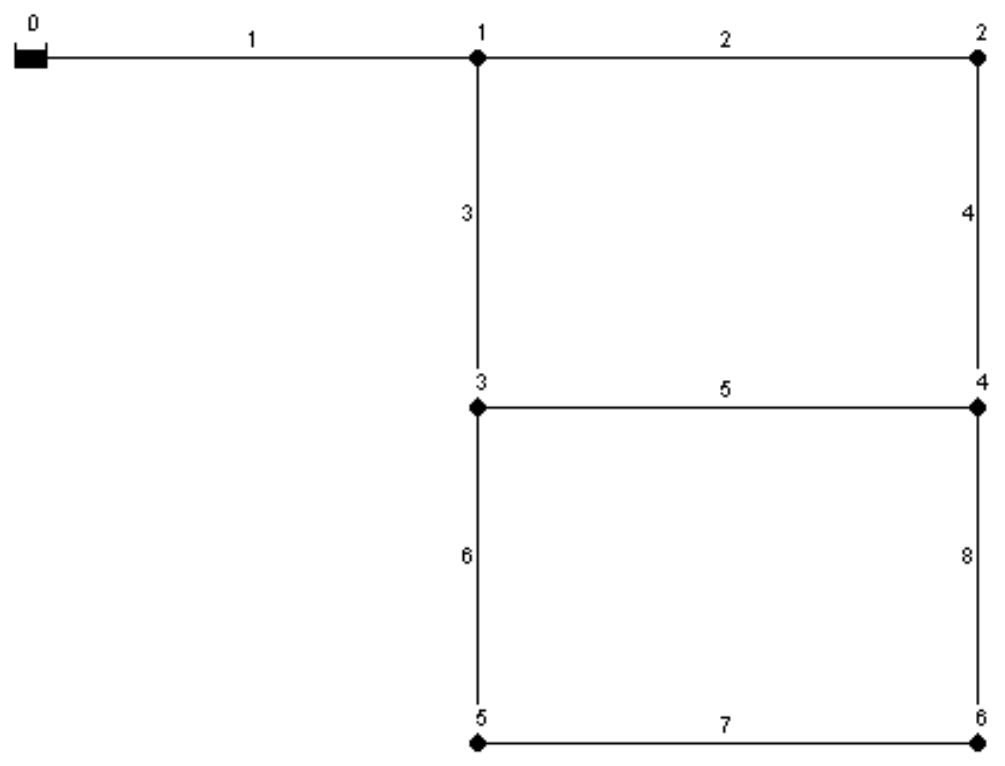

\begin{tabular}{|c|c|c|}
\hline Node & $\begin{array}{c}\text { Elevation } \\
(\mathrm{m})\end{array}$ & $\begin{array}{c}\text { Demand } \\
(\mathrm{l} / \mathrm{s})\end{array}$ \\
\hline 1 & 50 & 47.1 \\
\hline 2 & 50 & 47.1 \\
\hline 3 & 45 & 77.8 \\
\hline 4 & 45 & 47.1 \\
\hline 5 & 55 & 55.6 \\
\hline 6 & 55 & 88.9 \\
\hline
\end{tabular}

\begin{tabular}{|c|c|c|}
\hline Pipe & $\begin{array}{c}\text { Diameter } \\
(\mathrm{mm})\end{array}$ & $\mathrm{C}_{\mathrm{HW}}$ \\
\hline 1 & 500 & 140 \\
\hline 2 & 400 & 140 \\
\hline 3 & 400 & 140 \\
\hline 4 & 400 & 140 \\
\hline 5 & 250 & 140 \\
\hline 6 & 250 & 140 \\
\hline 7 & 250 & 140 \\
\hline 8 & 250 & 140 \\
\hline
\end{tabular}

Figure 1. Layout of the demonstration network

We considered three cases (Cases 1 to 3 ) to evaluate the models. Values of the bulk water reaction rate used previously in the literature were considered. Carrico and Singer (2009) and Helbling and Van Briesen (2009) summarized the reported values of bulk water reaction rates. The values range from 0.1 to $4.52 /$ day. For this work, a bulk water reaction rate of $1 /$ day was selected. A constant chlorine dose of $1 \mathrm{mg} / \mathrm{L}$ was applied at the source. A maximum THM concentration of $100 \mu \mathrm{g} / \mathrm{L}$ in the network has been assumed according to the Water Supply (Water Quality) Regulations (2010) in England and Wales. Also, a 5-minute water quality time step was specified. All simulations were carried out on a personal computer (Intel Core 2 Duo, 3.2 GHz, 3.21 GB RAM, Microsoft Windows XP operating system).

\section{Case 1}

In Case 1, the water level at the source was fixed at $90 \mathrm{~m}$ to ensure the network operates with sufficient pressure to satisfy all demands and achieve a 100\% demand satisfaction ratio (DSR). The first order, limited first order and zero order kinetic models were used to predict chlorine residual, THM concentration and water age respectively. Figure 2 shows that both EPANET-MSX and EPANET-PDX provide essentially identical results when pressure in the system is sufficient. Due to the multi-species capability of EPANET-MSX, this model can predict all the 3 species (water age, chlorine and THM) in a single simulation. EPANET-MSX required an average CPU time of 0.265 seconds to complete the analysis for a 24-hour operating cycle. On the other hand, to simulate a single species, EPANET-MSX required an average CPU time of 0.093 seconds. EPANET-PDX, however, which is a single species model, performed the same analysis by simulating only one species at a time. To carry out the water age analysis for a 24-hour period of operation, EPANET-PDX required an average CPU time of 0.038 seconds. This is almost 2.5 times faster than EPANET-MSX.

\section{Case 2}

In case 2 pressure deficient conditions were created by reducing the source water level from $90 \mathrm{~m}$ to $65 \mathrm{~m}$ and $60 \mathrm{~m}$ in Stages I and II respectively. This resulted in the network satisfying only $70 \%$ and $56 \%$ of the total demand in Stages I and II respectively. Cases 1 and 2 are identical in all other respects. For modelling the pressure deficient network, the head below which $Q n_{i}=0$ was taken as the nodal elevation while the head above which $Q n_{i}=Q n_{i}^{r e q}$ was taken as the elevation plus the 
minimum required residual head of $15 \mathrm{~m}$. Default values for $\alpha_{i}$ and $\beta_{i}$ were obtained using Equations 6 and 7 respectively.

Rather unexpectedly, EPANET-MSX provided identical water quality results to Case 1 (in which there is enough pressure to satisfy all demands). In practical terms, a pressuredeficient network cannot satisfy demands in full. In this regard, EPANET-MSX results are unrealistic. This limitation is attributable to the underlying DDA modelling approach. EPANET-PDX that has PDA functionality provided different water quality results that reflected the actual pressure in the network (Figure 3). Figure 3 shows that when the pressure in the system decreases, the THM concentration and water age increase while the chlorine residual decreases. This evidently reflects the fact that when the pressure in the network is low, the flow will correspondingly be low and the hydraulic residence time (water age) will be greater. An increase in residence time will enable the THM concentration to increase and the chlorine concentration to decrease. It is noted that low pressure conditions produced significant changes at distant nodes such as Nodes 5 and 6 as illustrated in Figure 3.

\section{Case 3}

In Case 3 the parallel first order model for chlorine decay and THM production was used. The source water level was fixed at 90 $\mathrm{m}$ and thus the network was operating under normal pressure conditions with $100 \%$ DSR. The multispecies capability of EPANETMSX allows simulation of the rapidly and slowly reacting components of the chlorine and THM. It should be noted, however, that EPANET-PDX cannot perform these simulations owing to its single species attribute.

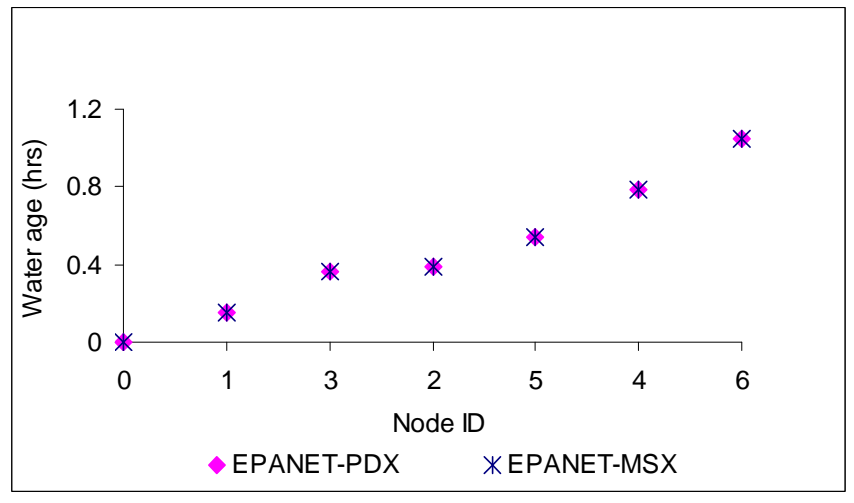

(a)

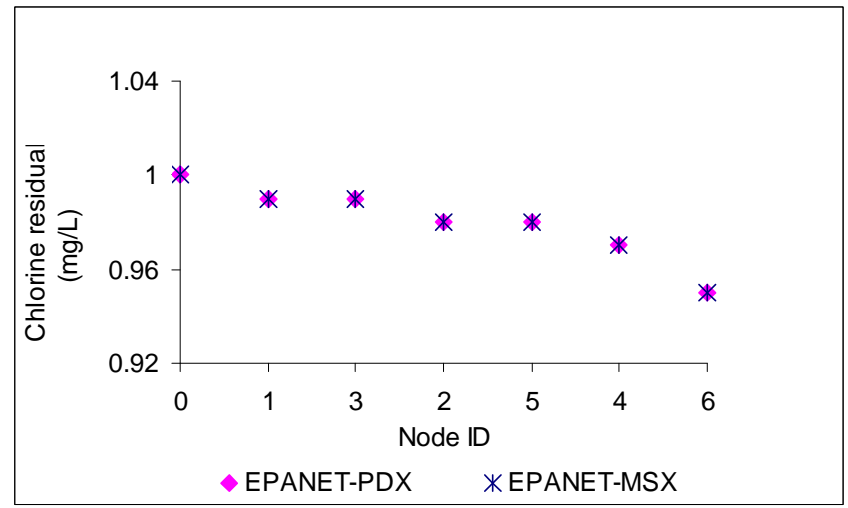

(b)

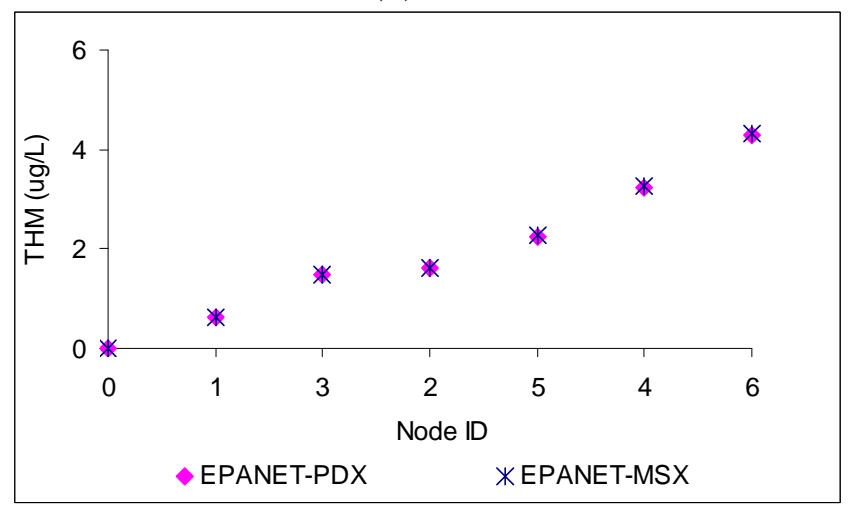

(c)

Figure 2. Comparison of water quality results for normal pressure conditions

For simulation of chlorine decay, Helbling and Van Briesen (2009) developed general-purpose empirical equations relating the initial concentration to each parameter of the parallel first order model. Accordingly we used $F=0.87, k_{1}=0.31 / \mathrm{hr}$ and $k_{2}=0.02 / \mathrm{hr}$. Sohn et al. (2004) developed empirical equations for estimating the parameters for THM production; we used $A=16$ and $\mathrm{B}=$ 34.7. 
It should be noted that EPANET-MSX can simulate all the species involved using the different kinetic models in a single simulation run. To simulate the seven species (chlorine decay, THM, water age, and the rapidly and slowly reacting fractions of chlorine and THM) at once, EPANETMSX required an average CPU time of 0.609 seconds. This is approximately 16 times the CPU time required by EPANET-PDX to simulate a single species. The chlorine residual and THM concentrations predicted using the parallel first order model have been compared with the values derived previously using the first order (Figure 4) and limited first order (Figure 5) models. Slight differences between the models can be observed in the results shown in each of Figures 4 and 5.

Although there seems to be reasonable agreement between these alternative formulations, in practice more accurate calibration of the two candidate models may be considered, which may involve fieldwork and laboratory tests. The main objective herein, however, is to demonstrate that EPANET-MSX can model both the chlorine residual and THM concentrations using parallel kinetic models whereas EPANETPDX cannot. Conversely EPANET-MSX cannot simulate subnormal pressure conditions realistically while EPANET-PDX does so seamlessly.

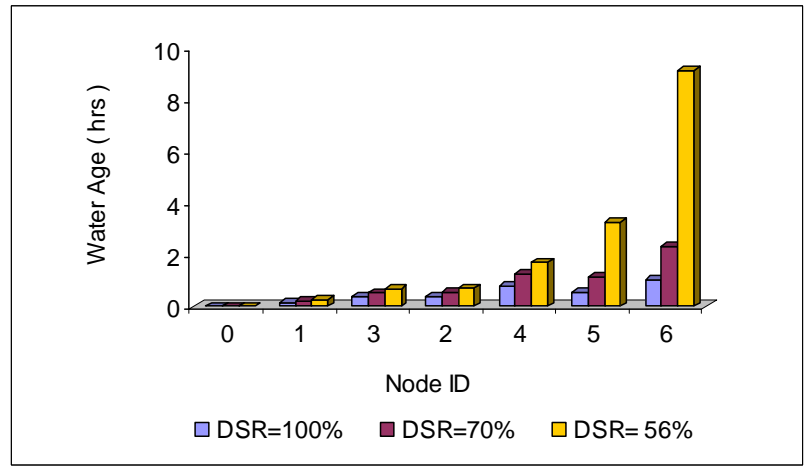

(a)

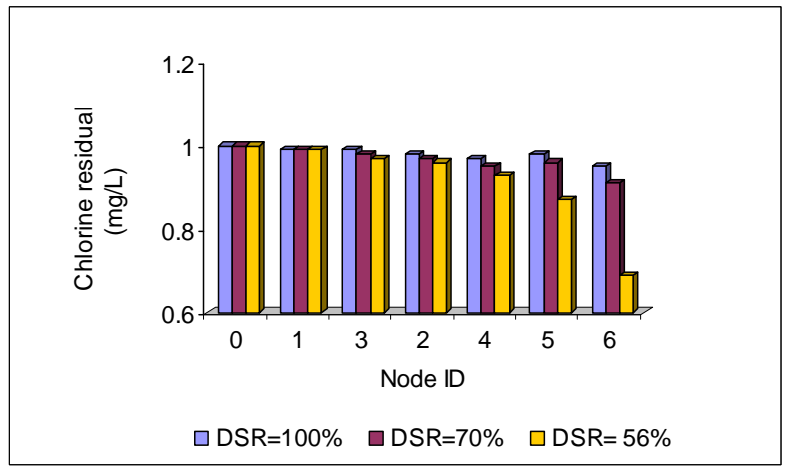

(b)

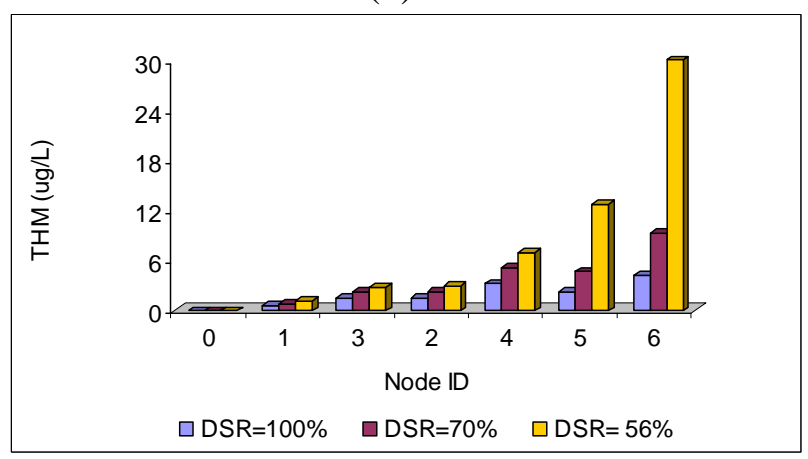

(c)

Figure 3. EPANET-PDX water quality results for normal and subnormal pressures

\section{CONCLUSIONS}

In this paper, the multiple species and pressure dependent EPANET extensions have been assessed. EPANET-MSX can model multiple interacting species but it is limited to networks operating under normal pressure conditions. On the other hand, EPANET-PDX, which is a pressure dependent model, can simulate both normal and pressure deficient networks seamlessly. However, the model is restricted to the dynamics of just a single species. This precludes simulation of reactions between two or more species accurately. The respective shortcomings of the two models provide inspiration to develop a holistic multi-species pressure dependent water quality model that can more accurately simulate the reactions between multiple species for appropriate and timely decision-making. The research described in this article is still in progress. 


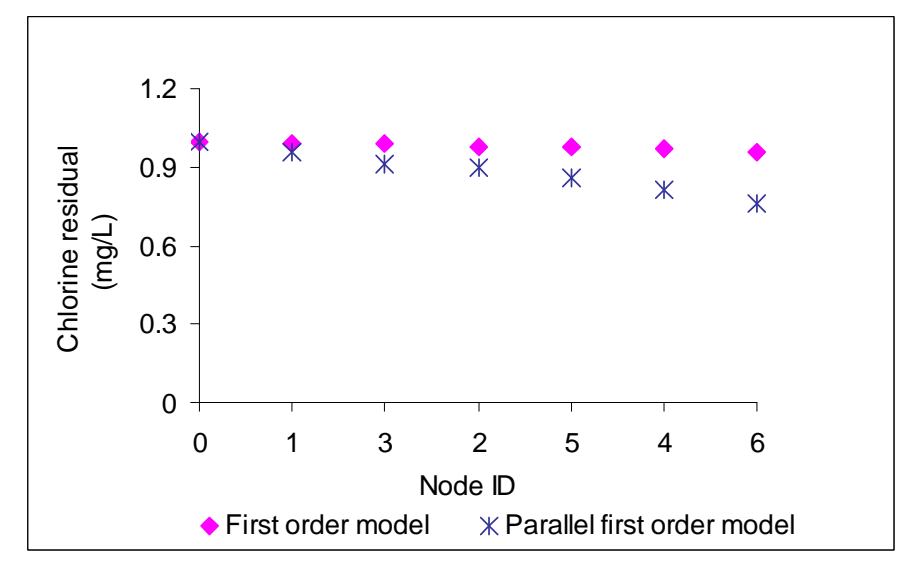

Figure 4. EPANET-MSX chlorine residuals under normal pressure

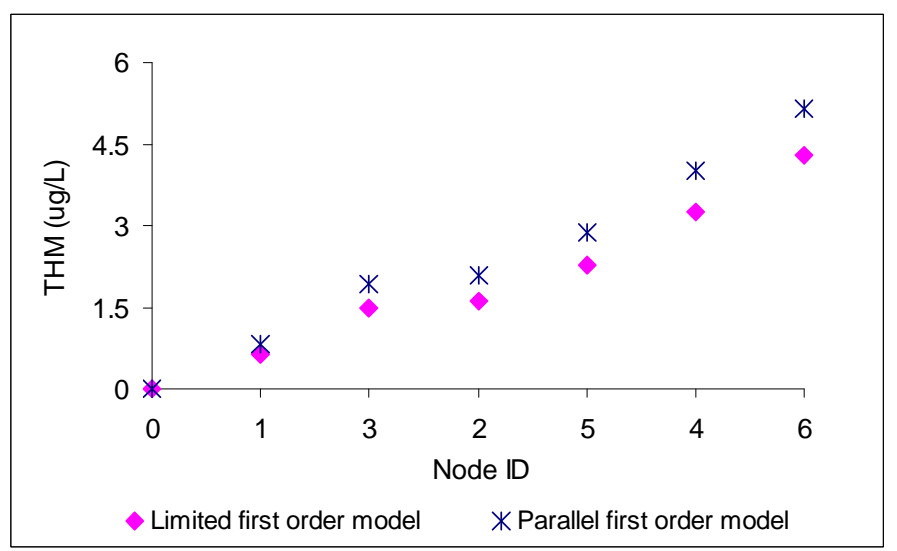

Figure 5. EPANET-MSX THM concentrations under normal pressure

\section{ACKNOWLEDGEMENT}

The authors are grateful to the British Government (Overseas Research Students Awards Scheme) and the University of Strathclyde for funding the first and third authors' PhD programmes. This project was funded in part by the UK Engineering and Physical Sciences Research Council (EPSRC Grant Reference EP/G055564/1).

\section{REFERENCES}

Carrico B. and Singer P.C. (2009). Impact of booster chlorination on chlorine decay and THM production: simulated analysis. J. Environmental Engineering, 135(10), 928-935.

Chandapillai J. (1999). Realistic simulation of water distribution system. J. Transportation Engineering. 117 (2), 258-263.

Clark R.M. and Haught R.C. (2005). Characterizing pipe wall demand: implications for water quality modelling. J. Water Resources Planning and Management, 31(3), 208-217.

Fujiwara O. and Ganesharajah T. (1993). Reliability assessment of water supply systems with storage and distribution networks. J. Water Resources Research, 29(8), 2917-2924. 
Gupta R. and Bhave P. R. (1996). Comparison of methods for predicting deficient network performance. Journal of Water Resources Planning and Management, 122 (3), 214-217.

Helbling D.E. and Van Briesen J.M. (2009). Modelling residual chlorine response to a microbial contamination event in drinking water distribution systems. J. Environmental Engineering, 135 (10), 918-927.

Rodriguez M.J., Serodes J.B. and Levallois P. (2004). Behaviour of trihalomethanes and haloacetic acids in a drinking water distribution system. Water Research, 38 (20), 4367-4382.

Rossman L.A., Boulos P.F. and Altman T. (1993). Discrete volume element method for network water quality models. Journal of Water Resources Planning and Management, 119 (5), 505-517.

Rossman L.A. (2000). EPANET 2 users manual, Water Supply and Water Resources Division, National Risk Management Research Laboratory, Office of Research and Development, U.S. Environmental Protection Agency, Cincinnati, Ohio.

Siew C. and Tanyimboh T.T. (2011). Practical application of the head dependent gradient method for water distribution networks. Water Science and Technology: Water Supply, 11(4), 444-450.

Siew C. and Tanyimboh T.T. (2012). Pressure-dependent EPANET extension. Water Resources Management, 26(6), 1477-1498.

Shang F., Uber J.G. and Rossman L.A. (2008). Modelling reaction and transport of multiple species in water distribution systems. Journal of Environmental Science and Technology, 42(3), 808-814.

Sohn J., Amy G., Cho J., Lee Y., and Yoon Y. (2004). Disinfectant decay and disinfection byproducts formation model development: chlorination and ozonation by-products. Water Research, 38, 2461-2478.

Tanyimboh T. T., Burd R., Burrows R. and Tabesh M. (1999). Modelling and reliability analysis of water distribution systems. Water Science and Technology 39 (4), 249-255.

Tanyimboh T.T. and Templeman A.B. (2010). Seamless pressure-deficient water distribution system model. Journal of Water Management, 163 (8), 389-396.

Water, England and Wales (2010). The Water Supply (Water Quality) Regulations, Statutory Instruments, No. 994 (W.99), Stationery Office, London, UK.

Wu Z.Y., Wang R.H., Walski T.M., Yang S.Y., Bowdlers D. and Baggett C.C. (2009). Extended global-gradient algorithm for pressure-dependent water distribution analysis. Journal of Water Resources Planning and Management, 135(1), 1-13. 